\title{
VIABILIDADE ECONÔMICA DO CULTIVO DE CARPA COMUM (Cyprinus carpio Linnaeus, 1758) EM MONOCULTIVO EM DENSIDADES DIFERENTES
}

\author{
Economical viability of the common carp (Cyprinus carpio Linnaeus, 1758) \\ cultivation in monocultivations in different densities
}

\begin{abstract}
Álvaro Graeff ${ }^{1}$
\section{RESUMO}

Objetivou-se com esta pesquisa estudar o efeito econômico do povoamento de alevinos I de carpa comum (Cyprinus carpio LINNAEUS, 1758) com densidade diferenciada e com alimentação artificial e adubação química. O delineamento foi inteiramente casualizado com três tratamentos $\left(1 ; 0,5\right.$ e 0,33 peixes $\left./ \mathrm{m}^{2}\right)$ avaliados ao longo do tempo e três repetições. Os peixes foram estocados com peso e comprimento médios de 2,40 g e 5,35; 5,40 e 5,39 cm, respectivamente, nos tratamentos, e com 45 dias de idade. As dietas foram formuladas com $27 \%$ de PB e $2925 \mathrm{Kcal} / \mathrm{Kg}$ de ração, sendo fornecidas diariamente na quantidade de $3 \%$ do peso vivo. Os resultados para o comprimento e peso dos peixes foram 25,68; 28,66 e 31,21 cm e 255,00; 424,00 e 519,66 g, respectivamente. A analise econômica foi estudada por meio de planilha de custo. Na produção total, os resultados foram 1882; 1513 e 1367 $\mathrm{kg} /$ hectare em um ciclo. Em condições similares, pode-se recomendar zootecnicamente todas as densidades estudadas e, economicamente, a lucratividade da atividade é crescente com o aumento da densidade, havendo diminuição do custo médio ou unitário.
\end{abstract}

Termos para indexação: Carpa comum, Cyprinus carpio, densidade, engorda, econômico.

\section{ABSTRACT}

This research aimed at studying the economical effect of common carp (Cyprinus carpio LINNAEUS, 1758) settling of fingerlings with differenciated density and with artificial feeding and chemical fertilization. The design was entirely randomized with three treatments $\left(1 ; 0.5\right.$ and 0.33 fishes $\left./ \mathrm{m}^{2}\right)$ evaluated along the time and 3 repetitions. The fish were stocked with medium weight and mediun length of $2.40 \mathrm{~g}$ and 5.35; 5.40 and $5.39 \mathrm{~cm}$, respectively, in the treatments, and 45 days old. The diets were formulated with $27 \% \mathrm{~PB}$ and $2.925 \mathrm{kcal} / \mathrm{kg}$ of ration, being given daily in the quantities of $3 \%$ of living weight. The results for the length and weight of the fishes were 25.68; 28.66 and $31.21 \mathrm{~cm}$ and $255.00 ; 424.00$ and $519.66 \mathrm{~g}$, respectively. The economical analisys was studied through cost planning. In the total production, the results were $1882 ; 1513$ and $1367 \mathrm{~kg} / \mathrm{ha}$ in a cycle. In similar conditions all the studied densities coud be zootechnically recommended. The economical profitability of the activity is growing with the increase of density and there is unitary or mediun cost decrease.

Index terms: Common carp, Cyprinus carpio, density, economical, fattering.

(Recebido para publicação em 17 de junho de 2002 e aprovado em 14 de janeiro de 2003)

\section{INTRODUÇÃO}

A necessidade de suprir a demanda por alevinos de carpa comum (Cyprinus carpio LINNAEUS, 1758) próximo ao mês de agosto (KREUZ e TAMASSIA, 1986) vem exigindo uma maior produção e tecnologia para desenvolvimento da engorda desses peixes. Para isso, são necessários métodos que diminuam os custos de produção (TAMASSIA e KREUZ, 1988). Sendo a temperatura um dos fatores importantes de estimulo à desova e alimentação das carpas (TAMASSIA, 1996; ZAVALACAMIN, 1996), as condições naturais da água (Figura 1) do Planalto Catarinense dificultam a propagação de carpas comuns e a engorda desses peixes antes de outubro, pois a temperatura das águas só ultrapassa os $18^{\circ} \mathrm{C}$ a partir da segunda quinzena de setembro (GRAEFF et al., 2001).

Uma das alternativas propostas para aumentar a oferta é a estocagem dos alevinos de origem tropical em altas densidades durante o inverno (HEPHER e PRUGININ, 1985; GRAEFF et al., 2001), procurandose, nesse caso, dispor de alevinos com tamanhos diferenciados, em quantidades suficientes e boas condições sanitárias no início da primavera, evitando altas taxas de mortalidade no período de inverno e adequando os custos com manejo e alimentação às baixas taxas de crescimento no inverno (BOLL et al., 1995). 


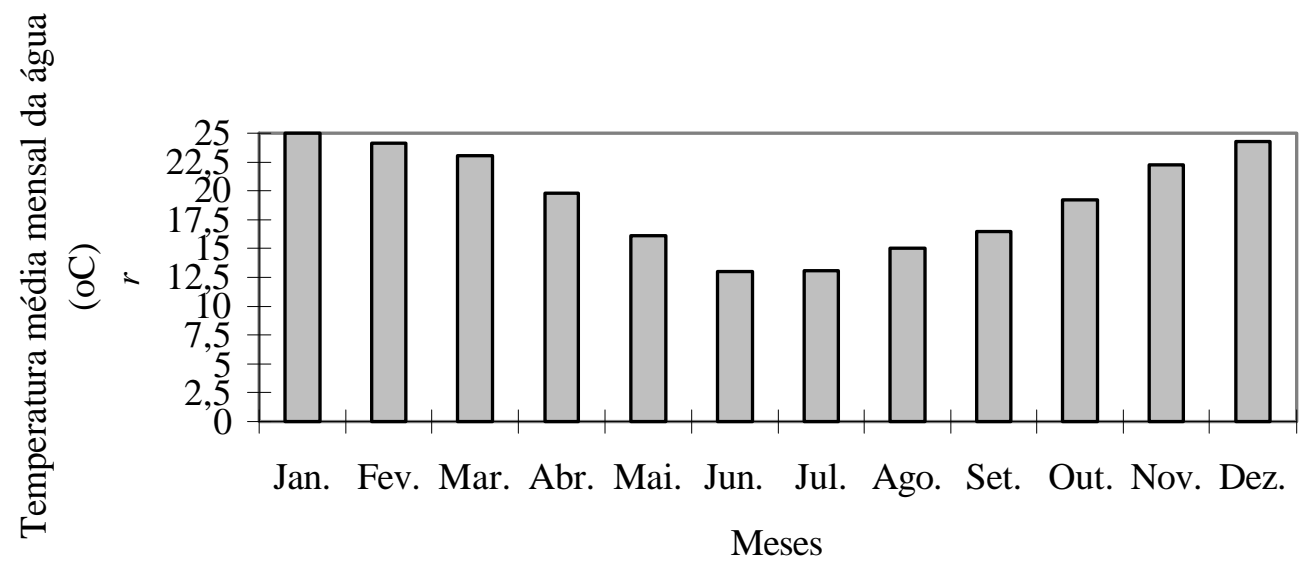

FIGURA 1 - Temperaturas médias mensais da água na Estação de Piscicultura de Caçador. Médias de cinco anos - 1993 a 1997.

Baseado no exposto acima, com o presente trabalho tem-se por objetivo a análise econômica da engorda de carpas comuns, em monocultivo em diferentes densidades, supondo a viabilidade/possibilidade da condução de dois ciclos de produção (um real e outro estimado) com suplementação artificial e adubação química no Planalto Catarinense, no período da primavera ao outono, com alevinos de origem de estocagem de inverno.

\section{MATERIAL E MÉTODOS}

A avaliação econômica foi realizada sobre o experimento conduzido em 120 dias de engorda (período de 22 de novembro de 1995 a 21 de março de 1996), no Posto de Piscicultura de Videira, Santa Catarina, sobre o desenvolvimento de alevinos seleção II, oriundos de 120 dias de estocagem de inverno na Estação de Piscicultura de Caçador/EPAGRI, Santa Catarina (GRAEFF et al., 2001).

Os tratamentos constam de três densidades de povoamento $\left(1,00 ; 0,50\right.$ e 0,33 peixes $\left./ \mathrm{m}^{2}\right)$ dispostos num delineamento inteiramente ao acaso com três repetições, avaliados a cada 30 dias para peso e comprimento.

A dieta foi formulada com produtos facilmente encontrados na região, dentro dos critérios para espécie $(27 \%$ de proteína bruta e $2925 \mathrm{kcal}$ de Energia metabolizável por quilograma de ração) e o sistema de produção (NRC, 1993) conforme a Tabela 1. A alimentação foi ofertada uma vez ao dia, na proporção de $3 \%$ do peso vivo. A quantidade de ração ofertada era reajustada a cada 30 dias, no momento das avaliações para peso e comprimento. Nesse momento, eram pesados com uma balança eletrônica com precisão de $0,01 \mathrm{~g}$, marca Mettler 4400-Delta Ranger e medidos aproximadamente $50 \%$ dos peixes de cada parcela com uma régua milimetrada.

Os viveiros foram adubados a cada 30 dias com $42,0 \mathrm{~kg}$ de Superfosfato Triplo com $46 \%$ de $\mathrm{P}_{2} \mathrm{O}_{5}$ e mais $75,0 \mathrm{~kg}$ de Uréia com $45 \%$ de $\mathrm{N} / \mathrm{hectare}$.

As verificações da temperatura da água foram realizadas mediante medições diárias com termômetro digital na superfície e a $0,80 \mathrm{~cm}$ de profundidade, às 9 horas e às 15 horas, quando também era oferecida a ração. Para avaliações da temperatura ambiente, utilizou-se um aparelho de corda, marca Wilh-Lambrech/Gmbh Gottingern. O acompanhamento da qualidade da água foi feito pelo Laboratório de Qualidade de Água/Epagri - Caçador; para as variáveis: $\mathrm{pH}$, oxigênio dissolvido, gás carbônico, dureza, alcalinidade, amônia, nitrato, turbidez, semanalmente. A transparência foi verificada uma vez por semana utilizando-se o disco de Secchi.

Ao término do experimento, foi realizada a análise econômica, tendo-se por base 1 hectare de produção de carpas comum (Cyprinus carpio LINNAEUS, 1758) em cada uma das três densidades estudadas, com dois ciclos de produção (um real e outro estimado), em uma planilha de custo.

Ciênc. agrotec., Lavras, v. 28, n. 3, p. 678-684, maio/jun., 2004 
TABELA 1 - Composição percentual da ração básica utilizada no experimento.

\begin{tabular}{|c|c|}
\hline Ingredientes & $\%$ \\
\hline Ração comercial $^{1}$ & 37,5 \\
\hline Farelo de Trigo & 21,7 \\
\hline Milho & 39,4 \\
\hline Óleo de Soja & 1,4 \\
\hline Total & 100,0 \\
\hline \multicolumn{2}{|l|}{ Composição } \\
\hline $\mathrm{EM}, \mathrm{Kcal} / \mathrm{kg}$ de ração & 2.925 \\
\hline Proteína bruta \% & 27,00 \\
\hline Cálcio \% & 2,55 \\
\hline Fósforo total \% & 1,31 \\
\hline Matéria fibrosa $\%$ & 8,99 \\
\hline Matéria mineral \% & 9,16 \\
\hline Extrato etéreo \% & 4,37 \\
\hline \multicolumn{2}{|c|}{$\begin{array}{l}{ }^{1} \text { Rações Truta Alevinos: Vit. A, 20.000UI; Vit. E, } \\
150 \text { mg; Vit. D, 4.000UI; Vit. K, } 5 \text { mg, Vit. } \text { B }_{6}, 14 \\
\text { mg; Vit. B12, } 60 \text { mcg; Tiamina } 20 \text { mg; Riboflavina } 40 \\
\text { mg; Pantotenato de Cálcio } 100 \mathrm{mg} \text {; Colina } 1.600 \text { mg; } \\
\text { Inositol } 300 \text { mg; Ac. Fólico } 6 \text { mg; Ac. Nicotinico } 140 \\
\text { mg; Biotina 0,2 mg } \\
\text { Níveis de Garantia (\%): Proteína Bruta (min) 50,00, } \\
\text { Extrato Etereo (min) 7,00, Matéria Fibrosa (max) } \\
\text { 4,00, Matéria Mineral (max) 19,5, Cálcio (max) 6,70, } \\
\text { Fósforo (min) 2,20 }\end{array}$} \\
\hline
\end{tabular}

\section{RESULTADOS E DISCUSSÃO}

\section{Avaliação Zootécnica}

O incremento do peso e comprimento médio dos peixes e biomassa final total durante a execução do experimento apresentou um resultado final zootecnicamente satisfatório, conforme a Tabela 2, já apresentado por Graeff e Pruner (2000), os quais afirmam que, dependendo do destino dado ao peixe, os maiores para filés ou in natura e os menores para conservas e fishburgues, qualquer densidade mostraria resultado significativo. Dentro do ponto de vista preconizado por Valentine (1972), isso é maximizar o rendimento, com verticalização da curva de crescimento dos peixes, favorecendo um rápido giro de capital e economia de alimento (VALENTINE, 1972).

A conversão alimentar aparente nas densidades de 1,00; 0,50 e 0,33 foi de 1,34; 1,05 e 1,23 e a sobrevivência 73,$78 ; 71,35$ e $75,21 \%$, respectivamente, estavam dentro do esperado para os experimentos da região.

Quanto ao uso de adubação química, o resultado foi eficaz para o aumento da biomassa total em complementação à ração oferecida, reafirmando a conclusão de Graeff e Pruner (1999), em que foi observado e identificado o aumento da biomassa natural por microscopia ótica.

A temperatura da água durante o período experimental (Tabela 3) manteve-se entre 20,4 e $21,8^{\circ} \mathrm{C}$, no período da manhã, ficando a média do período em $21,1^{\circ} \mathrm{C}$. No período da tarde, oscilou entre 24,6 e $25,2^{\circ} \mathrm{C}$ e a média ficou em $24,7^{\circ} \mathrm{C}$. Nota-se que as temperaturas foram inferiores às que afirmaram Arrignon (1979) e Makinouchi (1980): "o melhor crescimento das carpas se dá entre $24,0^{\circ}$ a $28,0^{\circ} \mathrm{C}$ ", fato que aparentemente não trouxe prejuízo ao crescimento dos alevinos. A temperatura média ambiente durante o experimento oscilou entre $25,7 \mathrm{e}$ $24,4^{\circ} \mathrm{C}$, ficando a média em $25,4^{\circ} \mathrm{C}$, normal para a média histórica da região.

TABELA 2 - Peso médio inicial e final, comprimento médio inicial e final, biomassa total nas três densidade (GRAEFF e PRUNER, 2000) na fase de engorda durante o verão.

\begin{tabular}{cccccc}
\hline \multirow{2}{*}{ Densidade } & \multicolumn{2}{c}{ Peso médio $(\mathbf{g})$} & \multicolumn{2}{c}{ Comprimento médio (cm) } & Biomassa Total(Kg)/Período \\
& Inicial & Final & Inicial & Final & 1881 \\
\hline 1,00 & 2,40 & 255,0 & 5,37 & 25,68 & 1512 \\
0,50 & 2,40 & 424,0 & 5,40 & 28,66 & 1302 \\
0,33 & 2,40 & 519,6 & 5,39 & 31,21 & \\
\hline
\end{tabular}

Ciênc. agrotec., Lavras, v. 28, n. 3, p. 678-684, maio/jun., 2004 
Os valores do $\mathrm{pH}, \mathrm{O}_{2} \mathrm{D}, \mathrm{CO}_{2}$, dureza, amônia, nitrito (Tabela 3), segundo Reid e Wood (1976), Huet (1978), Arrignon (1979), Lukowicz (1982), Ordog e Nunes (1988), Castagnolli (1992) e Arana (1997) estavam dentro do preconizado e são considerados normais para as criações de peixes.

A alcalinidade, pelo contrário, manteve-se entre 37,20 a 42,50 mg/L de $\mathrm{CaCO}_{3}$, dentro do nível recomendado por Boyd (1976) e Tavares (1994), que é de 30 a $300 \mathrm{mg} / \mathrm{L}$

A transparência (Tabela 3) permaneceu, durante todo período experimental, entre 24,0 e $50,0 \mathrm{~cm}$ de altura, indicando boa densidade de plâncton (TAVARES, 1994). A turbidez, que está diretamente correlacionada à transparência, permaneceu entre 9,40 e 23,22 NTU. Isso é consequiência do cultivo praticado no experimento, no qual há a presença de argilas colóidais, matéria orgânica dissolvida ou mesmo do plâncton (TAVARES, 1994).

\section{Avaliação Econômica}

Com a pesquisa em piscicultura objetiva-se não só melhorar a taxa de conversão alimentar, atingindo-se maior produtividade, mas também maximizar o rendimento, com verticalização da curva de crescimento dos peixes, favorecendo um rápido giro de capital e economia de alimento (VALENTINE, 1972).
Na Tabela 4 é apresentada uma planilha utilizada para a obtenção do custo de produção em um sistema de produção de 120 dias com densidades diferenciadas. Anteriormente, já foi comentado sobre o povoamento, peso médio inicial e final, taxa de sobrevivência. Na Tabela 5 são sumarizados os custos de manutenção do açude e sua depreciação em 20 anos, representando um custo fixo de $\mathrm{R} \$ 802,50$. O uso intensivo de adubos químicos, rações, alevinos e mão-de-obra (alimentação diária e despesca) representam um custo variável de $\mathrm{R} \$ 2.929,12 ; 3.141,56$ e 4.101,64, nas densidades de $3.500,5.000$ e 10.000 peixes por hectare respectivamente, em um ciclo de produção de 120 dias, possibilitando a realização de dois ciclos por ano. $\mathrm{O}$ custo total (custo fixo + custo variável) corresponde a um valor por quilo de peixe produzido de $\mathrm{R} \$ 1,36 ; 1,30$ e 1,30, nas densidades de 3.500 a 10.000 peixes/ha, respectivamente. $\mathrm{O}$ valor recebido pelos produtores da região, que vendem os peixes de 200 a $300 \mathrm{~g}$ para conservas e "fishburgers", e os maiores que $450 \mathrm{~g}$ para o processamento no frigorifico ou pesque-pague, foi $\mathrm{R} \$ 1,50$ o quilo. A diferença entre o custo total e a receita com os peixes proporcionou um lucro de $\mathrm{R} \$$ 369,38; 594,94 e 741,86 por hectare em dois ciclos, nas densidades de 3.500, 5.000 e 10.000 peixes/hectare, respectivamente. Portanto, a rentabilidade de se investir em tecnologia em alta densidade faz com que essa prática seja recomendável.

TABELA 3 - Média dos parâmetros limnológicos da água nas unidades experimentais em cada período do experimento de três densidade (GRAEFF e PRUNER, 2000) na fase de engorda durante o verão.

\begin{tabular}{lccccc}
\hline \multicolumn{1}{c}{ Parâmetros Limnológicos } & Dezembro & Janeiro & Fevereiro & Março & Média \\
\hline pH (potencial hidrogeniônico) & 7,50 & 6,84 & 6,63 & 7,20 & 7,04 \\
Oxigênio dissolvido (mg/L) & 8,40 & 8,86 & 8,55 & 8,85 & 8,66 \\
Gaz Carbônico (mg/L) & 10,00 & 11,20 & 12,00 & 11,00 & 11,05 \\
Dureza total (mg/L CaCO 3 ) & 42,00 & 45,60 & 43,00 & 52,50 & 45,77 \\
Alcalinidade (mg/L CaCO 3 ) & 40,00 & 37,20 & 42,50 & 42,50 & 40,55 \\
Amônia (mg/L) & 0,181 & 0,169 & 0,063 & 0,098 & 0,127 \\
Nitrito (mg/L) & 0,131 & 0,121 & 0,093 & 0,090 & 0,108 \\
Transparência (cm) & 45,00 & 24,00 & 39,75 & 50,00 & 39,68 \\
Turbidez (ntu) & 9,40 & 14,12 & 18,10 & 23,22 & 16,21 \\
Temperatura da água $(09 \mathrm{~h})^{\circ} \mathrm{C}$ & 20,4 & 21,3 & 21,1 & 21,8 & 21,1 \\
Temperatura da água $(15 \mathrm{~h}){ }^{\circ} \mathrm{C}$ & 24,6 & 25,2 & 24,6 & 24,7 & 24,7 \\
Temperatura ambiente ${ }^{\circ} \mathrm{C}$ & 24,4 & 25,6 & 26,1 & 25,7 & 25,4 \\
\hline
\end{tabular}


TABELA 4 - Planilha de custo de produção de carpa comum (Cyprinus carpio LINNAEUS, 1758) em 120 dias em três densidade (GRAEFF e PRUNER, 2000) na fase de engorda durante o verão.

\begin{tabular}{|c|c|c|c|c|}
\hline \multirow{2}{*}{$\begin{array}{c}\text { Item } \\
\text { Alevinos I/povoamento }\end{array}$} & \multirow{2}{*}{$\begin{array}{c}\text { Unidade } \\
\text { unidade/ha }\end{array}$} & \multicolumn{3}{|c|}{ Densidade de Alevino /hectare } \\
\hline & & 3500 & 5000 & 10000 \\
\hline Peso dos alevinos I & $\mathrm{g}$ & 2,40 & 2,40 & 2,40 \\
\hline Taxa de sobrevivência & $\%$ & 75,21 & 71,35 & 73,78 \\
\hline Ciclo de engorda & Dias & 120 & 120 & 120 \\
\hline Peixes & unidade/ha & 2633 & 3568 & 7378 \\
\hline Peso na despesca & g & 519 & 424 & 255 \\
\hline Produção & $\mathrm{Kg} / \mathrm{ha} / \mathrm{safra}$ & 1367 & 1513 & 1882 \\
\hline Uso de ração & $\%$ biomassa/dia & 3 & 3 & 3 \\
\hline Adubo - Uréia & kg/ha/mês & 65 & 65 & 65 \\
\hline Adubo - Super Triplo (ST) & $\mathrm{kg} / \mathrm{ha} / \mathrm{mês}$ & 34 & 34 & 34 \\
\hline Custo saco de Uréia & $50 \mathrm{~kg}$ & 27,50 & 27,50 & 27,50 \\
\hline Custo saco de ST & $50 \mathrm{~kg}$ & 31,50 & 31,50 & 31,50 \\
\hline Taxa de uso do adubo & $\%$ & 100 & 100 & 100 \\
\hline Necessidade de Uréia & $\mathrm{kg} / \mathrm{ha}$ & 260 & 260 & 260 \\
\hline Necessidade de ST & $\mathrm{kg} / \mathrm{ha}$ & 136 & 136 & 136 \\
\hline Custo do adubo/despesca & $\mathrm{R} \$$ & 228,68 & 228,68 & 228,68 \\
\hline Biomassa média mês 1 & $\mathrm{~kg} / \mathrm{ha}$ & 8,4 & 12,0 & 24,00 \\
\hline Biomassa média mês 2 & $\mathrm{~kg} / \mathrm{ha}$ & 290 & 335 & 424 \\
\hline Biomassa média mês 3 & $\mathrm{~kg} / \mathrm{ha}$ & 853 & 844 & 1220 \\
\hline Biomassa média mês 4 & $\mathrm{~kg} / \mathrm{ha}$ & 1317 & 1410 & 1820 \\
\hline Necessidade ração mês 1 & $\mathrm{~kg} / \mathrm{ha}$ & 8 & 11 & 22 \\
\hline Necessidade ração mês 2 & $\mathrm{~kg} / \mathrm{ha}$ & 261 & 302 & 382 \\
\hline Necessidade ração mês 3 & $\mathrm{~kg} / \mathrm{ha}$ & 768 & 760 & 1098 \\
\hline Necessidade ração mês 4 & $\mathrm{~kg} / \mathrm{ha}$ & 1185 & 1270 & 1638 \\
\hline Total de ração & $\mathrm{Kg} / \mathrm{ha}$ & 2.222 & 2.343 & 3.140 \\
\hline Preço da ração & $\mathrm{R} \$ / \mathrm{kg}$ & 0,32 & 0,32 & 0,32 \\
\hline Custo da ração & $\mathrm{R} \$$ & 711,04 & 749,76 & 1004,80 \\
\hline Alevino Seleção I - milheiro & $\mathrm{R} \$$ & 45,00 & 45,00 & 45,00 \\
\hline Salário Mínimo & $\mathrm{R} \$ / \mathrm{mês}$ & 180,00 & 180,00 & 180,00 \\
\hline Máquinas & $\mathrm{R} \$ / \mathrm{ha}$ & $4.400,00$ & $4.400,00$ & $4.400,00$ \\
\hline Monge & $\mathrm{R} \$ / \mathrm{ha}$ & 600,00 & 600,00 & 600,00 \\
\hline Custo construção do viveiro & $\mathrm{R} \$ / \mathrm{ha}$ & $5.000,00$ & $5.000,00$ & $5.000,00$ \\
\hline Custo da terra & $\mathrm{R} \$ / \mathrm{ha}$ & $1.500,00$ & $1.500,00$ & $1.500,00$ \\
\hline Custo total do viveiro & $\mathrm{R} \$ / \mathrm{ha}$ & $6.500,00$ & $6.500,00$ & $6.500,00$ \\
\hline
\end{tabular}

Ciênc. agrotec., Lavras, v. 28, n. 3, p. 678-684, maio/jun., 2004 
TABELA 5 - Custo de manutenção e lucratividade da produção de carpa (Cyprinus carpio LINNAEUS, 1758) em três densidade (GRAEFF e PRUNER, 2000) na fase de engorda durante o verão em dois cultivos de 120 dias.

\begin{tabular}{|c|c|c|c|c|c|}
\hline \multirow{2}{*}{ Item } & \multirow{2}{*}{ Unidade } & \multirow{2}{*}{$\begin{array}{l}\text { Coeficiente } \\
\text { Técnico }\end{array}$} & \multicolumn{3}{|c|}{ Densidade Alevinos/ha } \\
\hline & & & 3500 & 5000 & 10000 \\
\hline Depreciação viveiro & anos & 20 & 250,00 & 250,00 & 250,00 \\
\hline Juros & $\%$ aa & 6 & & & \\
\hline Terra & $\mathrm{R} \$ / \mathrm{ha}$ & & 90,00 & 90,00 & 90,00 \\
\hline viveiro & $\mathrm{R} \$ / \mathrm{ha}$ & & 300,00 & 300,00 & 300,00 \\
\hline Manutenção viveiro & $\%$ & 1,5 & 97,50 & 97,50 & 97,50 \\
\hline Despesas eventuais & $\%$ & 1,0 & 65,00 & 65,00 & 65,00 \\
\hline Custo fixo & $\mathrm{R} \$ / \mathrm{ha}$ & & 802,50 & 802,50 & 802,50 \\
\hline Custo da adubação & $\mathrm{R} \$ / \mathrm{ha}$ & & 457,36 & 457,36 & 457,36 \\
\hline Custo da ração & $\mathrm{R} \$ / \mathrm{ha}$ & & $1.422,08$ & $1.499,52$ & $2.009,60$ \\
\hline \multicolumn{6}{|l|}{ Custo da mão de obra } \\
\hline Alimentação & horas/ano & 720 & 661,22 & 661,22 & 661,22 \\
\hline Despesca & horas/ano & 80 & 73,46 & 73,46 & 73,46 \\
\hline Custo dos alevinos II & $\mathrm{R} \$ / \mathrm{mil} / \mathrm{ha}$ & 90 & 315,00 & 450,00 & 900,00 \\
\hline Custo variável & $\mathrm{R} \$ / \mathrm{ha}$ & & $2.929,12$ & $3.141,56$ & $4.101,64$ \\
\hline Custo Total & $\mathrm{R} \$ / \mathrm{ha}$ & & $3.731,62$ & $3.944,06$ & $4.904,14$ \\
\hline Custo médio Peixe/kg & $\mathrm{R} \$ / \mathrm{kg}$ & & 1,36 & 1,30 & 1,30 \\
\hline Receita com Peixes & $\mathrm{R} \$ / \mathrm{Kg}$ & 1,50 & $4.101,00$ & $4.539,00$ & $5.646,00$ \\
\hline Lucro com os Peixes & $\mathrm{R} \$ / \mathrm{ha}$ & & 369,38 & 594,94 & 741,86 \\
\hline
\end{tabular}

\section{CONCLUSÕES}

Com o aumento da densidade da população, a produção final é influenciada proporcionalmente a esse aumento.

$\mathrm{O}$ aumento da densidade populacional diminui o ganho de peso individual e o crescimento individual dos peixes.

O povoamento com densidades crescentes de até 10.000 alevinos de carpa comum/ha em dois ciclos de produção na região é economicamente viá- vel, sendo o crescimento da maior parte de sua população satisfatório.

A lucratividade da atividade é crescente com o aumento da densidade, havendo diminuição do custo médio ou unitário do custo do quilo do peixe produzido.

\section{REFERÊNCIAS BIBLIOGRÁFICAS}

ARANA, L. V. Princípios químicos de qualidade da água em aquicultura: uma revisão para peixes e camarões. Florianópolis: UFSC. 1997. 166 p. 
ARRIGNON, J. Ecologia y piscicultura de águas dulces. Madrid: Mundi Prensa, 1979. 365 p.

BOLL, M. G.; SATO, H.; AMARAL JÚNIOR, H. Resultados preliminares de método alternativo do manejo da tilápia, Oreochromis niloticus, no período de inverno em regiões de Clima Subtropical. In: ENCONTRO SUL BRASILEIRO DE AQUICULTURA, 3.; ENCONTRO RIOGRANDENSE DE TÉCNICOS EM AQUICULTURA, 6., 1995, Ibiruba/RS. Anais... Porto Alegre: UFRGS, 1995. p. 88-93.

BOYD, C. E. Limme requirements and application in fishponds. Aqconf, Kyoto, v. 176, n. 13, p. 6, 1976.

CASTAGNOLLI, N. Piscicultura de água doce. Jaboticabal: FUNEP, 1992. 189 p.

GRAEFF, A.; KREUZ, C. L.; PRUNER, E. N.; SPENGLER, M. M. Viabilidade econômica de estocagem de alevinos de carpa comum (Cyprinus carpio var. specularis) no inverno em alta densidade. Revista Brasileira de Zootecnia, v. 30, n. 4, p. 1150-1158, 2001.

GRAEFF, A.; PRUNER, E. N. Efeito da densidade de estocagem na produtividade final em carpas Cyprinus carpio LINNAEUS, 1758 (var. specularis) na fase de engorda: período inverno. Ciência e Agrotecnologia, Lavras, v. 23, n. 4, p. 959-968, 1999.

GRAEFF, A.; PRUNER, E. N. Efeito da densidade de povoamento na produtividade final em carpas (Cyprinus carpio var. specularis) em fase de engorda, durante o verão. Revista Brasileira de Zootecnia, Viçosa, v. 29, n. 3, p. 639-645, 2000.

HEPHER, B.; PRUGININ, Y. Cultivo de peces comerciales, baseado en las experiencias de las granjas piscicolas en Israel. México: Limusa, 1985. 316 p.

HUET, M. Tratado de piscicultura. Madrid: Mundi Prensa, 1978. 745 p.

KREUZ, C. L.; TAMASSIA, S. T. J. Viabilidade econômica da produção comercial de alevinos de carpa comum a nível de produtor rural. Florianópolis: EMPASC, 1986. 17 p. (Documentos, 65).

LUKOWICZ, M. V. Intensive carp (Cyprinus carpio L.) is rearing in a farm pond in southern Germany and its effects on water quality. Aquaculture Engineers, [S.1.], v. 1, n. 2, p. 121-137, 1982.

MAKINOUCHI, S. Criação de carpas em água parada. Informe Agropecuário, Belo Horizonte, v. 6, n. 67, p. 30-47, 1980.

NATIONAL RESEARCH COUNCIL. Nutrient requirements of fish. Washington, 1993. $116 \mathrm{p}$.

ORDOG, V.; NUNES, Z. M. P. Sensibilidade de peixes a amônia livre. In: SIMPÓSIO LATINO AMERICANO DE AQUICULTURA, 6.; SIMPÓSIO BRASILEIRO DE AQUICUlTURA, 5., 1988, Florianópolis/SC. Anais... Florianópolis: ABRAq, 1988. p. 169-174.

REID, G. K.; WOOD, R. D. Ecology of Island waters and estuaries. New York: D. Van Nostrand, 1976. 485 p.

TAMASSIA, S. T. J. Carpa comum (Cyprinus carpio): produção de alevinos. Florianópolis: EPAGRI, 1996. 75 p. (Boletim Técnico, 76).

TAMASSIA, S. T. J.; KREUZ, C. L. Viabilidade econômica da recria de carpas em Santa Catarina: estudo de casos. In: SIMPÓSIO LATINO AMERICANO DE AQUICULTURA, 6.; SIMPÓSIO BRASILEIRO DE AQUICULTURA, 5., 1988, Florianópolis/SC. Anais... Florianópolis: ABRAq, 1988. p. 603-607.

TAVARES, L. H. S. Limnologia aplicada a aquicultura. Jaboticabal: FUNEP, 1994. 70 p.

VALENTINE, J. W. Conceptual models of ecosystem evolution. In: _ Models in paleobiology. San Francisco: Freeman, 1972. p. 192-215.

ZAVALA-CAMIN, L. A. Introdução ao estudo sobre alimentação natural em peixes. Maringá: EDUEM, 1996. 129 p. 